\section{ON THE RELATION OF PARTIAL RETENTION OF URINE TO ITS DECOMPOSITION WITHIN THE BLADDER.}

BY WILLIAM S. SAVORY, F.R.S., SURGEON TO ST. BARTHOLOMEW'S HOSPITAL.

THE opinion is, I believe, current, it is certainly general in our books and elsewhere, that inability to empty the bladder completely is a common cause of chronic inflamma. tion of the viscus, because a portion of stagnant urine is retained until it is decomposed, and so becomes an irritant. In this way, by confining some of the urine until it becomes decomposed, it is stated that all forms of obstruction to its exit produce chronic cystitis. Now, is not this opinion that residual urine thus remains to decompose based on a misconception of the actual facts, or at allevents of the cause, in such a case? Is it not assumed that the urine which remains behind is a fixed quantity, and therefore stagnant? Is it not overlooked that, although the bladder may be never emptied, yet that, inasmuch as a large portion of the urine it contains is frequently leaving it to be replaced by another portion which is continually entering it, the same portion of urine does not necessarily remain long enough in the bladder to become, of itself, the source of the mischief that ensues? Of course urine may remain long enough in the bladder to decompose, for no one needs to be reminded of the fetid urine reeking of ammonia in chronic cystitis; and it admits of demonstration that urine which enters the bladder in a healthy state is very rapidly changed by the disordered secretion of that organ. But this need not depend simply on retention, either total or partial. Micturition may be frequent, and the bladder may be frequently quite emptied, and yet the urine that flows may be putrid; or urine may be retained for a very long period, and yet when it escapes there may be no evidence of decomposition. In many cases, for example, where a person is confined to the recumbent position, the bladder is never able to expel the whole of its contents, and yet this state of things may continue for weeks without the residual urine showing any sign of decomposition; and in other cases, where it may even become necessary to draw off the urine in this enforced position of the patient, the bladder, in all probability, never becomes completely emptied, and yet, at least for a long while, the urine passes without change. Therefore, even if all reasoning oa the matter be excluded, it eannot, in the face of these facts, be accepted that as a rule residual urine is to be regarded as the source of the mischief that is so often associated with it. No doubt the longer any given quantity of urine is retained in the bladder, when the causes of its decomposition are rife there, the farther will its decompusition proceed. But the duration of the retention of any given portion is by no means represented by the amount of residual urine after each act of micturition. The quantity of urine which enters and escapes from the bladder daily must rather be considered. Just as the water drawn off from a cistern gives no evidence of being stale or changed, although the reservoir is never emptied, because the whole of its contents are nevertheless rapidly renewed.

If healthy urine is passed into a bottle capable of holding, say, two pints, and the bottle as it becomes filled is from time to time only partially emptied, so that always about balf a pint be left behind, the urine in the bottle, even after months, will still retain an acid reaction. It does not become ammoniacal or acquire the characters of the urine in chronic inflammation of the bladder. And in this experiment the circumstances, so far as ordinary decomposition of stale urine is concerned, are, on the whole, far more favour. able to change; for although the temperature of the bottle may not be so high as that of the bladder, yet air is frequently and freely admitted, and of course it is always abundantly present. If the changes by which urine becomes ammoniacal are due only to long. continued partial retention, how is the negative result of this experiment to be explained?

Yet, although I venture to think that the prevailing opinion in this respect is not well founded, I am indeed very far from saying, or even intending to suggest, that there is no evil in partial retention. On the contrary, it is clear enough that the presence of residual urine implies some delay in the escape of a portion of it, and therefore in a corresponding degree favours the advance of decomposition. But this, I need not remark, is a very different view from that which regards partial retention as a common startingpoint of eystitis and the further evils which hence ensue. But then the fact remains that these two evils-residual urine, the result of partial retention, and chronic cystitisare very commonly associated. Can this be explained? The direct and immediate cause of the decomposition of urine in the bladder, which enters it in a healthy state, is the presence and action of a morbid secretion of the mucous membrane. Practically the changes of urine in the bladder are dependent on the action of unhealthy mucus, and the degree of decom. position, although of course affected by the time the urine remains there, is yet chiefly determined by the character of the diseased secretion which mingles with it. Even under the conditions of health it is the mucus present in the urine which mainly determines the decomposition of urea, as the result of the simple experiment of filtering fresh urine shows, and when mucus is morbid it is far more destructive. Now, this disordered secretion must be the product of an unhealthy membrane; and while there is but little difficulty in understanding how, when the texture of its lining membrane is altered, the bladder is hampered in its action, and its full and complete contraction impeded, there is hardly less difficulty in believing that a full and complete contraction of it from time to time is an essential condition of a healthy mucous membrane, and of a due secretion and free escape of healthy mucus. Therefore, the conditions under which the bladder is only partia'ly emptied are oftentimes the very conditions under which unhealthy, and therefore highly mischievous, nucus is brought in contact with the urine there.

\section{ON A NEW TEST FOR ALBUMEN IN URINE}

BY WM. ROBERTS, M.D., F.R.S., PHYSICIAN TO THE MANCHESTER ROYAL INFIRMARY.

WHEN an albuminous urine is treated with a saturated solution of common salt, not the slightest reaction takes place; but if the brine be slightly acidulated with hydrochloric acid, the albumen is thrown down as a dense white cloud. This reaction constitutes a most delicate test for albumen in the urine. The best degree of acidulation for this purpose is obtained with about 5 per cent. of the dilute hydrochloric acid of the Pharmacopoia. A little more or a little less acid makes no appreciable difference in the sensitiveness of the test. Common salt dissolves in about two and a half times its weight of water at $60^{\circ} \mathrm{F}$, and increase of temperature does not sensibly increase its solubility. The salt of commerce is al ways more or less dirty, and the solution requires filtration to fit it for use as a test. The salt solution should be fully saturated, otherwise the observer is apt to be led into error. In preparing the test with our common English measures the readiest plan is to mix a fluid ounce of dilute hydrocbloric acid with a pint of water, and to saturate this with common salt, and filter. Dilute hydrochloric acid may be replaced by dilute sulphuric, dilute nitric, or dilute phosphoric acid. All these acids are of the same saturating strength in the British Pharmacopoia, and all of them yield with saturated salt solution an equally sensitive reagent for albumen. Even acetic acid may be used, but the delicacy of the tf st in that case is not quite so great as when it is prepared with one of the mineral acids. The method of apulying the brine test is similar to that followed with nitric acid. A portion of the suspected urine is placed in a testtube, the test-tube is then held very much aslant, and the salt solution is allowed to trickle along the sides of the tube to the bottom, so that it may form a distinct layer below the urine. If albumen be present, a wbite cloudy zone appears at the junction of the two fluids. Or the proceeding may be reversed. The salt solution may be first introduced into the test-tube, and then the urine added with the same precautions as before, so as to obtain two distinct layers, one abnve the other, in the test-tube. It is important to be aware that the precipitation of albumen by acidulated brine is not due to a true coagnlation. In this respect the 
brine test differs from nitric acid and boiling. In the two latter cases the albumen is transformed into the issoluble modification, which is known as "congulated albumen." But when albumen is thrown down from urine by acidulated brine, the precipitate is not insoluble; on the contrary, it is redissnlved by free addition of water, or even by free addition of the albuminous urine itself. It is there- fore essential to the efficieut application of the test that the salt solution should be in excess at the point of expected reaction. This end is obviously secured in the abovedescribed methods of testiog. It may also be secured by addiog to the suspected uriue a volume of the salt solution at least equal to that of the wine in the test-tuhe. If this pint be not attended to the test is unreliable. For instance, if acidulated brine be added drop by drop to an albuwinous urine, and the mixture shaken up after each addition, the first fow drops either occasion no turbidity whatsuever or the turbidity produced disappears on shaking. But when by successive additions the quantity of brine approaches to or surpasses the volume of urine operated on the turbidity remains permanent. In point of delicacy the salt test stands on a par with nitric acid. The minutest trace of albumen detectable in the urine by nitric acid is al=o detectable with equal ea-e by acidulated brine. In hichcoloured urines the brine test is distinctly superior. In this class of urines nitric acid produces a deepening of the tint, with, often, a disengagement of gas, which interteres with the sensitiveness of the reaction, but the brine test neither alters the tint nor causes disengagement of gas. On the other hand, I think that nitric acid gives a better idea of the quantity of albumen present by the density of the white cloud produced thau does the brine test. In addition to albumen, acidulated brine precipitaus peptones, which are sometimes present in urine; so that occasionally a slight cloudiness is produced by the salt solution where nitric acid and boiling (which do not precipitate peptones) produce no reaction. This distinction in the action of the brine test may hereafter lead to interesting information. In dense urines, highly charged with urates (but not containing albumen), the addition of nitric acid sometimes throws down the amorphous urates in the furm of thick white clouds, and it is necessary to apply heat to distinguìsh with certainty the c'oudiness so produced trom cloudiness due to albumen. The salt test dises not throw down the urates in this way. It is well known that the urines of patients who are taking large doses of resinous substances (snch as the resin of copaiba), although fiee from albumen, yield a cloudiness with nitric acid in the cold, but if the urine be previously made hot, nitric acid produces no such reaction. This difference serves to distinguish cloudiness due to resin from cloudiness due to albumen. The brine test also produces a cloudiness in resinous urines, and the reaction occurs whether the urine be hot or cold. To aroid the fallacy thereby arising, all that is necessary is to add an excess of the urine which is being te-ted. If the cloudiness be due to albumen it disappears on such addition, but if it be due to resin, the cloudiness does not disappear on the addition of more urine. Une of the chief advantages of the salt test is its incorrosive character. It does not stain nor burn holes in garments and carpets, nor fleck the hands with yellow spots. The use of it makes it possible to arrange a pocket-case for urine testing that shall not be a terror to the wearer. From this point of view the suhstitution of the salt solution for nitric acid will be a real boon to practitioners. ${ }^{1}$ The salt test has this additional convenience-that it enables us to test successively for albumen and sugar on one and the same sample of urine. The suspected urine is first tested for albumen with the salt solution, and then Fehling's solution, or, still better, a pellet of the solid Fehling's test sent out by Cooper is added, and heat applied. After boiling a few seconds the absence or presence of agar is ascertained. The admixture of the brine in no way interferes with the copper reaction, in case sugar should exist in the urine. Manchester.

I bave carried about with me for some months past a little pocketcase (which is only a stiff-backed cigar-case) which I wave found a useful and safe clinical companion. It contains a book of litmus papers; narrow corked phial filled with acidulated brine; a test-tube charged with Cooper's pellets of the solid Febling's test, guarded with an indiarubber stopper; and, lastly, an empty test-tube, also provided with a cork. This compact arrangement furnishes the means of ascertaining the reaction of the urine, and of testing it in the most delicate manner for albumen and sugar. The empty test-tube also serves to curry home a specimen of the urine for further and more minute examinution The " " peecimen of the urine for further and more minute examination. "The Cooper, chemist, 26, Oxford-street, London.

\section{VOLUMETRIC ESTIMATE OF ALBUIEX IN URINE}

\section{BY GUY NEVILLE STEPHEN, M.R.C.S.}

In the wards, and in private practice, the method most commonly employed for detecting the presence and estimating the amount of albumen in urine is the application of heat, the result beiog aided and verified by the addition of a few drops of nitric acid. As a simple test, this method, though not absolutely free from objection, either on the score of chemical accuracy or ease of application-a matter of consicterable importance in private practice,-is yet, when applied wich ordinary care, sufficiently reliable and search. ing to warrant its popularity, and prevent one receiving as anything more than scientifically interesting the various other albumen tests that have been and are from time to time proposed. As a volumetric test, however, applied by precipi. tating the albumen, allowing it to cool and subside, and then noting the proportion of the albumen which has collected to the rest of the fluid in the test-tube, and reading it off as a fraction, it is at best but a very rough method, with numerous sources of error, and, as commonly employed, gires most in. accurate and deceptive results, due partly to inberent causes, and partly to want of care in throwing down the whole of the albumen and allowiug it to thoroughly cool and subside. Such as it is, nevertheless, this method is even more universally employed in the estimate of albumen than are the same reagents, heat and nitric acid, as a simple test of its presence; but probably less, because general approval is accorded to it, than because of the other methods generally known none has a claim to be substituted for it -Esbacli's test being, if more accurate, much more tedious, while the actual weighing of the albumen is, from the readiness with which albumen absorbs moisture, a process that taxes the resources of even a skilled chemist. Since, therefore, we are at present in possession of no easy, rapid, and trust. worthy method of estimating albumen, every fresh idea and suggestion on the subject should receive a thorough clinieal trial before being relegated to the chemical laboratory; and this his not yei been accorded either in England or abroad to a methud suguested by Tauret of Troyes as far back as 1872. The basls of this method is the presipitation of alloumen by the double iodide of mercury and potassium, and it is equally applicable both for testing for and estimat ing albumen. The manner of procedure is extremely simple, and in practice the testing and estimation maybe done at the same time, but for the sake of descriptive clearness the testing will here be taken first.

Testing.-For this all that is required is a solution of the double iodide of mercury and notassium and some acetic acid. The urine to be tested first must bs reudered distinctly acid by the help of some of the acetrc acid, and then a few drops of the solution added to it. If a tbumen be present a white opaque flocculent precipitate immediately forms, which on the one hand requires no verification like that firmed by the application of heat to only slightly albuminous uriue, and on the other is not, like the precipitate of nitric acid, liable to be redissolved by adding too much of the reagent. In point of delicacy, moreover, the test is superior to either of these, as the precipitate will form in the presence of as small a quantity of albumen as tive centigrammes to a litre of water, a result which is only equalled by the very uncertain phenic acid test, and by beating with sulphate of soda. To the practitioner, too, who wishes to test urine for al bumen by the bedside of his patient, it is a very distmct advantage to be able to dispense with test-tube, spirit lamp, and strong nitric acid, and to carry with him nothing but two small phials, and perbars some litnus papers. It is scarcely ntcessary to enter into possible causes of confusion, as the only case in which the reagent under discussion itself gives rise to any other than al'suminous precipitate is in the urine of patients who are taking alkaloids, when a precipitate may be formed which, however, is soluble either by heat or by the addition of alcohol; while the errors that may be entailed by the enployment of the acetic acid, together with the means of avoiding them, are already for the most part well known. In urine loaded with urates a precipitate of uric acid may 